\title{
Genetic Variability Assessment of Tossa Jute (Corchorus olitorius L.) Genotypes Using Morpho-Agronomic Traits
}

\section{Arju Miah ${ }^{1 *}$, Nihar Ranjan Saha ${ }^{2}$, AKM Shahadat Hossain ${ }^{1}$, Md Younus $\mathrm{Ali}^{3}$ and Amit Kumar Basunia ${ }^{4}$}

${ }^{1}$ Genetic Resources and Seed Division, Bangladesh Jute Research Institute, Manik Mia Avenue, Dhaka, Bangladesh

${ }^{2}$ Department of Biotechnology, Bangladesh Agricultural University, Bangladesh

${ }^{3}$ Fibre Quality Improvement Division, Bangladesh Jute Research Institute, Manik

Mia Avenue, Dhaka, Bangladesh

${ }^{4}$ Department of Horticulture, Bangladesh Agricultural University, Mymensingh,

Bangladesh

*Corresponding Author: Arju Miah, Genetic Resources and Seed Division, Bangladesh Jute Research Institute, Manik Mia Avenue, Dhaka, Bangladesh.

DOI: 10.31080/ASAG.2020.04.0865
Received: June 21, 2020

Published: June 30, 2020

(C) All rights are reserved by Arju Miah., et al.

\section{Abstract}

Forty accessions with three check varieties of tossa jute (Corchorus olitorius L.) were characterized on the basis of morpho-agronomic traits in genetic variability at Jute Agriculture Experiment Station, Manikganj during kharif season in 2016. The accessions were characterized for twenty-two morpho-agronomic attributes as per Corchorus descriptor in order to select superior genotypes for the genetic improvement of jute. Considerable ranges of variability were observed in stem colour, petiole colour, stipule colour, plant technical height, base diameter, dry fibre weight and dry stick weight. Considering the major yield contributing characters accession no. 1039, 1115, 1124, 1234 and1245 performed better in most of the cases than the control varieties 0-9897 0-3820 and BJRI Tossa Pat-4 (0-72).

Keywords: Corchorus; Variability; Genotype

\section{Introduction}

Jute (Corchorus spp.) is a natural fibre crop and is second in the world after cotton in terms of global production, consumption and availability [1]. It is cultivated mostly in Eastern India, Bangladesh, Nepal and some South East Asian countries and some parts of Africa [2]. Entire life-cycle of jute from cultivation to usage disposal, it is friendly to the environment and produces no toxic material [3]. Jute is still the leading cash crop and an important tradable and foreign exchange-earning (4.82\%) commodity of Bangladesh. The jute fibre is used, as a raw material, for several products like hessians, sacs, and carpet backings. The two species of the genus Corchorus, which are cultivated as jute crop include $C$. capsularis (white jute) and $C$. olitorius (tossa jute), each with $2 \mathrm{n}=14$, are cultivated in our country, but Corchorus olitorius is predominantly grown by the farmers. Due to increase the environmental consciousness the demand of natural fibre increases rapidly through the world. To meet this increasing demand there is an urgent need to redesign the ongoing breeding strategies to improve both the yield and quality of jute. Information on the nature and magnitude of genetic variability for the desired characters in the base material and interrelationship among them is useful in breeding for high 
yield [4]. It is therefore, essential to understand the variability and diversity in the available germplasm of jute. Increased pressure has been exerted on jute scientists to develop varieties with high yield potential and superior grade fibre that meet the demands of the textile industries. The use of plant genetic resources in breeding research is largely dependent on the available information of their genetic variability. In this study forty accessions of tossa jute (Corchorus olitorius) germplasm collected from Gene Bank Department of Bangladesh Jute Research Institute were characterized by morpho agronomic traits to find out the superior genotypes by using the Corchorus descriptor.

\section{Methodology}

The experiment conducted by sowing seeds on 9 April 2016. Forty entries along with the check varieties 0-9897, 0-3820 and BJRI Tossa Pat-4 (0-72) were taken to this study. Each accession was sown in 5 rows of $3 \mathrm{~m}$ length, spacing was $30 \mathrm{~cm}$ between rows, $5-6 \mathrm{~cm}$ between plants and $1 \mathrm{~m}$ between plots. Standard cultural and inter-cultural practices were followed. Pigmentation data on stem colour, leaf colour, vein colour, petiole colour, stipule colour, bud colour, and fruit colour were collected at 60 days after sowing and pre-bud stage. Plants were harvested at 120 days after sowing and post-harvest data were collected as per Corchorus Descriptor. Data were analyzed following standard statistical procedures.

\section{Results and Discussion}

The analysis of variance (ANOVA) for fibre yield and yield contributing components like plant height, base diameter, core diameter, dry stick weight etc. in tossa jute germplasm, is described in table 1. The ANOVA revealed significant difference as the source of variation, for the traits plant height $(\mathrm{m})$, base diameter $(\mathrm{cm})$, fibre weight (g/plant) and stick weight ( $\mathrm{g} / \mathrm{plant}$ ). All parameters are significantly different at $1 \%$ level of significance. The findings of the analysis of variance (ANOVA) for fibre yield components in tossa jute germplasm is similar to the findings of Roy., et al [5].

\begin{tabular}{|c|c|c|c|c|c|c|c|c|c|c|c|c|c|}
\hline $\begin{array}{l}\text { Source of } \\
\text { variation }\end{array}$ & df & $\begin{array}{c}\text { Tech ht } \\
\text { (m) }\end{array}$ & $\begin{array}{l}\text { Leaf Angl } \\
\text { (dg) }\end{array}$ & $\begin{array}{c}\text { Leaf Lnth } \\
\text { (cm) }\end{array}$ & $\begin{array}{c}\text { Leaf } \\
\text { Width } \\
\text { (cm) }\end{array}$ & $\begin{array}{l}\text { Petiol } \\
\text { Lnth } \\
\text { (cm) }\end{array}$ & Node No. & $\begin{array}{c}\text { Base Dia } \\
(\mathrm{mm})\end{array}$ & $\begin{array}{c}\text { Middle } \\
\text { Dia } \\
(\mathrm{mm})\end{array}$ & $\begin{array}{c}\text { Top Dia } \\
\text { (mm) }\end{array}$ & \begin{tabular}{|c|} 
Core \\
Dia \\
$(\mathrm{mm})$ \\
\end{tabular} & $\begin{array}{c}\text { Dry } \\
\text { Fibre } \\
\text { wt. (g) }\end{array}$ & $\begin{array}{c}\text { Dry Stick } \\
\text { wt. (g) }\end{array}$ \\
\hline & 2 & & & & 0.007 & 0.113 & 0.053 & 0.082 & 0.046 & 0.048 & 0.123 & 0.156 & \\
\hline Acces & 43 & $0.393 * *$ & $50.410^{* *}$ & $6.653^{* *}$ & $5.460^{* *}$ & $1.155^{* *}$ & $565.060^{* *}$ & $8.457^{* *}$ & $2.604^{* *}$ & $1.074^{* *}$ & $5.188^{* *}$ & $7.281^{* *}$ & $63.300^{* *}$ \\
\hline Error & 86 & 0.031 & 8.716 & 0.227 & 0.125 & 0.135 & 19.518 & 0.209 & 0.075 & 0.039 & 0.155 & 0.097 & 0.414 \\
\hline
\end{tabular}

Table 1: Analysis of variance (mean square).

**: Significant at $1 \%$ level of probability.

The mean performance of the major yield contributing characters and co-efficient of variation are presented in table 2. Pigmentation data on stem colour, leaf colour, vein colour, petiole colour, stipule colour, bud colour, and fruit colour are presented in table 3 . The plant technical height at harvest (120 days) ranged from 2.31 - 3.88m. The highest score was observed in accession 1039 (3.88 $\mathrm{m} /$ plant) followed by accessions no. 1124 (3.71 m/plant), 1115 (3.67 m/plant), 1234 (3.66 m/plant) and 1245 (3.62 m/ plant). Dry fibre weight ranged from $5.89-11.93 \mathrm{~g} / \mathrm{plant}$. The highest dry fibre weight was observed in accession 1039 (11.93 g/plant) followed by accessions 1124 (11.75 g/plant), 1234 (11.55 g/plant), 1115 (11.49 g/plant) and 1245 (1132 g/plant). Dry stick weight ranged from 15.25 - $33.87 \mathrm{~g} /$ plant. The highest score was recorded in accession no. 1039 (33.87 g/plant) followed by accession 1124 (33.23 g/plant), 1115 (32.95 g/plant), 1234 (32.89 g/plant) and
1245 (32.45 g/plant). Similar result was found in Annual Research Report [6] BJRI in different accession. Among the accessions characterized, five accessions viz. 1039, 1115, 1124, 1234 and 1245 performed better in most of the cases than the control varieties 0-9897, 0-3820 and BJRI Tossa Pat-4.

The phenotypic coefficient of variation (PCV) was found to be greater than the genotypic coefficient of variation (GCV) in case of all the characters (Table 4). The percentage (\%) of PCV of plant height 12.52 , base diameter 11.06 , dry fibre weight 16.11 and dry stick weight 17.14 and the percentage (\%) of GCV of plant height 11.17, base diameter 10.66 , dry fibre weight 15.79 and dry stick weight 16.97 was found in this study. The GCV and PCV were found to differ significantly for all the fibre yield components. This is in agreement with the findings of Sawarkar., et al [7]. The percentage 


\begin{tabular}{|c|c|c|c|c|c|c|c|c|c|c|c|c|}
\hline $\begin{array}{l}\text { Acc. } \\
\text { No. }\end{array}$ & $\begin{array}{c}\text { Tech ht } \\
\text { (m) }\end{array}$ & $\begin{array}{l}\text { Leaf } \\
\text { Angl } \\
\text { (dg) }\end{array}$ & $\begin{array}{c}\text { Leaf Lnth } \\
\text { (cm) }\end{array}$ & $\begin{array}{c}\text { Leaf } \\
\text { Width } \\
\text { (cm) }\end{array}$ & $\begin{array}{l}\text { Petiol } \\
\text { Lnth } \\
\text { (cm) }\end{array}$ & $\begin{array}{c}\text { Node } \\
\text { No. }\end{array}$ & $\begin{array}{c}\text { Base Dia } \\
(\mathrm{mm})\end{array}$ & $\begin{array}{c}\text { Middle } \\
\text { Dia } \\
(\mathrm{mm})\end{array}$ & $\begin{array}{c}\text { Top Dia } \\
\text { (mm) }\end{array}$ & $\begin{array}{c}\text { Core Dia } \\
(\mathrm{mm})\end{array}$ & $\begin{array}{c}\text { Dry Fibre } \\
\text { wt. (g) }\end{array}$ & $\begin{array}{c}\text { Dry Stick } \\
\text { wt. (g) }\end{array}$ \\
\hline 1032 & 2.95 & 50 & 15.36 & 7.25 & 5.95 & 85 & 15.69 & 8.98 & 3.92 & 13.87 & 9.67 & 27.64 \\
\hline 0-9897 & 3.08 & 50 & 15.21 & 8.21 & 6.58 & 90 & 15.75 & 9.42 & 4.11 & 4.58 & 10.48 & 29.25 \\
\hline 1033 & 3.12 & 48 & 16.58 & 7.35 & 6.25 & 92 & 15.69 & 8.79 & 4.15 & 14.55 & 10.76 & 29.47 \\
\hline 1034 & 2.69 & 54 & 14.55 & 5.15 & 5.48 & 60 & 12.67 & 7.79 & 3.87 & 12.54 & 7.45 & 20.38 \\
\hline 1036 & 2.98 & 52 & 15.32 & 5.54 & 5.84 & 87 & 14.95 & 7.75 & 3.98 & 13.74 & 9.85 & 26.85 \\
\hline 0-72 & 3.54 & 46 & 16.24 & 8.65 & 6.77 & 100 & 16.78 & 9.86 & 4.98 & 13.54 & 11.21 & 32.85 \\
\hline 1037 & 2.88 & 50 & 12.86 & 5.52 & 5.54 & 86 & 15.54 & 7.85 & 3.97 & 15.06 & 8.55 & 22.55 \\
\hline 1038 & 3.21 & 47 & 16.34 & 8.25 & 6.13 & 99 & 16.17 & 8.94 & 4.29 & 14.75 & 10.69 & 29.45 \\
\hline $0-3820$ & 3.26 & 44 & 16.21 & 8.34 & 6.67 & 95 & 16.59 & 8.98 & 4.38 & 14.89 & 10.86 & 27.57 \\
\hline 1039 & 3.88 & 40 & 17.21 & 9.12 & 7.95 & 112 & 17.85 & 10.75 & 5.92 & 15.96 & 11.93 & 33.87 \\
\hline 1111 & 2.78 & 50 & 14.25 & 5.75 & 5.65 & 65 & 12.58 & 7.75 & 3.89 & 12.51 & 7.89 & 20.54 \\
\hline 0-795 & 3.25 & 48 & 15.55 & 8.47 & 6.18 & 95 & 16.33 & 8.86 & 4.45 & 4.54 & 10.78 & 27.28 \\
\hline 1112 & 3.38 & 47 & 15.64 & 8.46 & 6.55 & 97 & 16.51 & 8.89 & 4.55 & 14.75 & 10.88 & 26.45 \\
\hline 1115 & 3.67 & 41 & 16.88 & 8.95 & 7.36 & 106 & 17.45 & 9.95 & 5.59 & 15.65 & 11.49 & 32.95 \\
\hline 1117 & 3.45 & 42 & 15.48 & 8.44 & 6.69 & 99 & 16.74 & 9.68 & 4.96 & 14.93 & 10.78 & 29.55 \\
\hline 1118 & 3.14 & 44 & 15.22 & 7.94 & 6.58 & 96 & 15.45 & 8.96 & 4.52 & 4.65 & 10.55 & 28.34 \\
\hline 1119 & 2.59 & 52 & 13.32 & 5.55 & 5.46 & 75 & 12.25 & 7.25 & 3.65 & 12.95 & 7.51 & 21.21 \\
\hline 1120 & 2.87 & 50 & 14.42 & 5.32 & 5.78 & 80 & 15.21 & 7.57 & 3.88 & 13.65 & 8.35 & 23.86 \\
\hline 1124 & 3.71 & 40 & 17.08 & 8.96 & 7.64 & 109 & 17.52 & 10.06 & 5.69 & 15.69 & 11.75 & 33.23 \\
\hline 1125 & 3.25 & 47 & 15.25 & 8.12 & 6.54 & 94 & 6.32 & 8.82 & 4.39 & 4.87 & 10.65 & 28.75 \\
\hline 1130 & 3.19 & 49 & 15.64 & 8.25 & 6.56 & 95 & 15.75 & 8.55 & 4.35 & 14.75 & 10.55 & 29.66 \\
\hline 1230 & 3.26 & 48 & 15.12 & 8.24 & 6.45 & 100 & 16.88 & 8.96 & 4.69 & 14.96 & 10.56 & 29.18 \\
\hline 1232 & 3.11 & 50 & 15.31 & 8.32 & 6.26 & 99 & 16.26 & 8.87 & 4.36 & 14.89 & 10.25 & 29.32 \\
\hline 1234 & 3.66 & 42 & 16.85 & 8.90 & 7.72 & 105 & 17.23 & 9.89 & 5.25 & 15.35 & 11.55 & 32.89 \\
\hline 1235 & 2.78 & 50 & 13.57 & 6.52 & 5.56 & 75 & 13.56 & 7.54 & 3.75 & 13.62 & 8.12 & 22.89 \\
\hline 1236 & 2.85 & 54 & 14.71 & 6.56 & 5.59 & 80 & 15.25 & 7.96 & 3.78 & 13.58 & 8.98 & 23.24 \\
\hline 1237 & 2.96 & 52 & 13.56 & 7.14 & 5.76 & 85 & 14.58 & 11.03 & 3.98 & 13.75 & 9.87 & 25.35 \\
\hline 1238 & 3.55 & 42 & 15.58 & 8.85 & 6.85 & 102 & 17.12 & 8.95 & 4.56 & 14.98 & 11.26 & 31.44 \\
\hline 1239 & 3.18 & 48 & 14.87 & 8.57 & 6.48 & 95 & 16.65 & 8.56 & 4.16 & 14.85 & 10.52 & 29.54 \\
\hline 1240 & 2.31 & 55 & 11.55 & 4.85 & 5.15 & 55 & 11.25 & 6.96 & 3.51 & 10.75 & 5.89 & 15.25 \\
\hline 1241 & 2.64 & 54 & 12.32 & 5.72 & 5.87 & 78 & 12.36 & 7.25 & 3.68 & 12.85 & 6.57 & 17.85 \\
\hline 1242 & 3.21 & 50 & 15.53 & 8.37 & 6.45 & 96 & 16.45 & 8.69 & 4.35 & 14.98 & 10.11 & 28.87 \\
\hline 1243 & 2.95 & 51 & 14.57 & 6.55 & 5.78 & 82 & 15.45 & 8.76 & 3.72 & 13.35 & 9.91 & 25.54 \\
\hline 1244 & 3.56 & 44 & 15.89 & 8.58 & 6.48 & 98 & 17.27 & 9.52 & 4.98 & 15.05 & 11.12 & 32.65 \\
\hline 1245 & 3.62 & 43 & 16.21 & 8.66 & 6.51 & 100 & 17.35 & 9.65 & 5.13 & 15.14 & 11.32 & 32.45 \\
\hline 1246 & 3.28 & 53 & 16.11 & 8.25 & 6.51 & 97 & 16.64 & 9.02 & 4.35 & 14.58 & 10.85 & 29.77 \\
\hline 1247 & 2.89 & 50 & 13.23 & 7.25 & 5.84 & 75 & 14.56 & 8.23 & 3.85 & 12.85 & 8.86 & 23.65 \\
\hline
\end{tabular}




\begin{tabular}{|l|c|c|c|c|c|c|c|c|c|c|c|c|}
\hline 1248 & 2.47 & 52 & 11.52 & 5.44 & 5.52 & 65 & 12.54 & 7.25 & 3.68 & 10.98 & 6.23 & 17.55 \\
\hline 1249 & 3.38 & 43 & 16.05 & 8.24 & 6.44 & 98 & 17.15 & 9.05 & 4.52 & 14.95 & 10.59 & 28.28 \\
\hline 1250 & 3.19 & 45 & 15.59 & 8.15 & 6.36 & 89 & 16.85 & 8.96 & 3.28 & 14.65 & 10.85 & 29.75 \\
\hline 1251 & 2.69 & 51 & 13.21 & 5.35 & 5.84 & 75 & 13.52 & 7.56 & 3.75 & 11.25 & 8.16 & 22.76 \\
\hline 1253 & 3.03 & 50 & 14.91 & 7.84 & 6.58 & 95 & 16.65 & 8.82 & 4.18 & 11.63 & 10.58 & 30.36 \\
\hline 1254 & 2.58 & 50 & 12.25 & 5.61 & 5.96 & 68 & 14.22 & 8.25 & 3.75 & 12.21 & 8.12 & 23.25 \\
\hline 1255 & 2.81 & 52 & 12.36 & 5.68 & 5.88 & 65 & 14.55 & 8.56 & 3.91 & 12.35 & 8.25 & 23.58 \\
\hline Range & $2.31-3.88$ & $40-55$ & $11.55-$ & $4.85-9.12$ & $5.15-$ & $55-$ & $11.25-$ & $6.96-$ & $3.51-$ & $10.75-$ & $5.89-11.93$ & $15.25-$ \\
& & 17.21 & & & 112 & 18.85 & 10.75 & 5.92 & 15.96 & \\
\hline Mean & 3.25 & 50.40 & 14.89 & 7.39 & 6.27 & 88.50 & 15.54 & 8.72 & 4.28 & 13.90 & 9.78 & 26.97 \\
\hline CV\% & 8.92 & 10.43 & 7.85 & 11.52 & 8.95 & 13.87 & 9.85 & 11.55 & 13.52 & 11.84 & 18.65 & 16.49 \\
\hline
\end{tabular}

Table 2: Range, mean and co-efficient of variation (CV \%) of twelve characters of forty Corchorus olitorius germplasm. *: Check Variety.

\begin{tabular}{|c|c|c|c|c|c|c|c|c|}
\hline Acc. No. & Stem color & Leaf color & Vein Color & Petiole color & Stipule & Stipule color & Bud color & Fruit Color \\
\hline 1032 & G & G & G & G & + & G & $\mathrm{Y}$ & B \\
\hline $0-9897$ & G & $G$ & G & $G$ & + & G & $\mathrm{Y}$ & $\mathrm{B}$ \\
\hline 1033 & $\mathrm{G} / \mathrm{R}$ & G & G & $\mathrm{G} / \mathrm{R}$ & + & $\mathrm{G} / \mathrm{R}$ & $\mathrm{Y}$ & $\mathrm{B}$ \\
\hline 1034 & G & G & $\mathrm{G}$ & $G$ & + & G & $\mathrm{Y}$ & $\mathrm{B}$ \\
\hline 1036 & G & $\mathrm{G}$ & G & G & + & G & $\mathrm{Y}$ & $\mathrm{B}$ \\
\hline $0-72$ & G & $G$ & $G$ & $G$ & + & $G$ & $\mathrm{Y}$ & $\mathrm{B}$ \\
\hline 1037 & $\mathrm{G}$ & $G$ & $\mathrm{G}$ & $G$ & + & $\mathrm{G}$ & $\mathrm{Y}$ & $\mathrm{B}$ \\
\hline 1038 & G & G & G & G & + & G & $\mathrm{Y}$ & $\mathrm{B}$ \\
\hline $0-3820$ & G & G & G & G & + & G & $\mathrm{Y}$ & $\mathrm{B}$ \\
\hline 1039 & $\mathrm{G} / \mathrm{R}$ & $G$ & $\mathrm{G}$ & $\mathrm{G} / \mathrm{R}$ & + & $\mathrm{G} / \mathrm{R}$ & $\mathrm{Y}$ & $\mathrm{B}$ \\
\hline 1111 & G & G & G & G & + & G & $\mathrm{Y}$ & $\mathrm{B}$ \\
\hline $0-795$ & $\mathrm{R}$ & G & $G$ & $\mathrm{G} / \mathrm{R}$ & + & $\mathrm{G} / \mathrm{R}$ & $\mathrm{Y}$ & $\mathrm{B}$ \\
\hline 1112 & G & $G$ & $G$ & G & + & $\mathrm{G}$ & $Y$ & B \\
\hline 1115 & G & G & $G$ & $G$ & + & $G$ & $\mathrm{Y}$ & B \\
\hline 1117 & $\mathrm{G}$ & $\mathrm{G}$ & $G$ & $G$ & + & $G$ & $Y$ & $\mathrm{~B}$ \\
\hline 1118 & $G$ & $G$ & $\mathrm{G}$ & $\mathrm{G}$ & + & G & $Y$ & $\mathrm{BB}$ \\
\hline 1119 & $G$ & $G$ & $G$ & $G$ & + & $G$ & $Y$ & $\mathrm{~B}$ \\
\hline 1120 & $\mathrm{G}$ & $\mathrm{G}$ & $\mathrm{G}$ & $\mathrm{G}$ & + & $\mathrm{G}$ & $\mathrm{Y}$ & B \\
\hline 1124 & $\mathrm{G} / \mathrm{R}$ & $G$ & $G$ & G/R & + & $\mathrm{G} / \mathrm{R}$ & $\mathrm{Y}$ & $B$ \\
\hline 1125 & $\mathrm{G} / \mathrm{R}$ & $G$ & $G$ & $\mathrm{G} / \mathrm{R}$ & + & $\mathrm{G} / \mathrm{R}$ & $\mathrm{Y}$ & $\mathrm{B}$ \\
\hline 1130 & G & $G$ & $G$ & $G$ & + & $G$ & $\mathrm{Y}$ & B \\
\hline 1230 & $\mathrm{G}$ & $G$ & G & G & + & G & $\mathrm{Y}$ & B \\
\hline 1232 & $\mathrm{G}$ & $G$ & $\mathrm{G}$ & $\mathrm{G}$ & + & $\mathrm{G}$ & $Y$ & B \\
\hline 1234 & G & $G$ & $\mathrm{G}$ & $\mathrm{G}$ & + & G & $Y$ & B \\
\hline 1235 & G & $G$ & $\mathrm{G}$ & $\mathrm{G}$ & + & G & $\mathrm{Y}$ & $\mathrm{B}$ \\
\hline 1236 & $G$ & $\mathrm{G}$ & $G$ & $G$ & + & G & $\mathrm{Y}$ & $\mathrm{B}$ \\
\hline 1237 & $\mathrm{G} / \mathrm{R}$ & $\mathrm{G}$ & $G$ & $G$ & + & $\mathrm{G} / \mathrm{R}$ & $\mathrm{Y}$ & $\mathrm{B}$ \\
\hline 1238 & $\mathrm{G} / \mathrm{R}$ & G & $G$ & $\mathrm{G} / \mathrm{R}$ & + & $\mathrm{G} / \mathrm{R}$ & $\mathrm{Y}$ & B \\
\hline
\end{tabular}




\begin{tabular}{|l|c|c|c|c|c|c|c|c|}
\hline 1239 & G & G & G & G & + & G & Y & B \\
\hline 1240 & G & G & G & G & + & G & Y & B \\
\hline 1241 & G & G & G & G & + & G & Y & B \\
\hline 1242 & G & G & G & G & + & G & Y & B \\
\hline 1243 & G & G & G & G & + & G & Y & B \\
\hline 1244 & G/R & G & G & G/R & + & G/R & Y & B \\
\hline 1245 & G & G & G & G & + & G & Y & B \\
\hline 1246 & G & G & G & G & + & G & Y & B \\
\hline 1247 & G & G & G & G & + & G & Y & B \\
\hline 1248 & G & G & G & G & + & G & Y & B \\
\hline 1249 & G & G & G & G & + & G & Y & B \\
\hline 1250 & G & G & G & G & + & G & Y & B \\
\hline 1251 & G & G & G & G & + & G & Y & B \\
\hline 1253 & G & G & G & G & + & G & Y & B \\
\hline 1254 & G & G & G & G & + & G & Y & B \\
\hline 1255 & G/R & G & G & G/R & + & G/R & Y & B \\
\hline
\end{tabular}

Table 3: Pigmentation of Corchorus olitorius germplasm along with check varieties 0-9897, 0-72, 0-795, and BJRI 0-3820. $\mathrm{G}=$ Green, $\mathrm{R}=$ Red, LR = Light Red, $\mathrm{Y}=$ Yellow, B = Brown, “+” = Present, * = Check Variety.

(\%) of heritability of plant height 79.56 , base diameter 92.94 , dry fibre weight 96.11 and dry stick weight 98.06 and the percentage (\%) of genetic advance (GA) of plant height 20.52, base diameter 21.18, dry fibre weight 31.89 and dry stick weight 34.62 was found in this study. The heritability and genetic advance (\% of mean) were also found to be high for all the traits and this is similar to the findings of Roy., et al. [8] who reported that higher heritability and genetic advance for fibre yield components of tossa jute.

\begin{tabular}{|c|c|c|c|c|c|c|c|c|c|c|c|}
\hline $\begin{array}{l}\text { SL. } \\
\text { No. }\end{array}$ & Characters & Minimum & Maximum & Mean & \begin{tabular}{|c|} 
Genotypic \\
variance \\
$\left(d^{2} \mathbf{g}\right)$ \\
\end{tabular} & $\begin{array}{c}\text { Phenotypic } \\
\text { variance } \\
\left(d^{2} p\right) \\
\end{array}$ & GCV (\%) & PCV (\%) & $\begin{array}{c}\text { Heritability } \\
\left(\mathbf{h}^{2} \mathbf{b}\right)\end{array}$ & GA & GA (\%) \\
\hline 1 & Tech ht (m) & 2.31 & 3.88 & 3.11 & 0.12 & 0.15 & 11.17 & 12.52 & 79.56 & 0.64 & 20.52 \\
\hline 2 & Leaf Angl (dg) & 40.00 & 55.00 & 48.18 & 13.90 & 22.61 & 7.74 & 9.87 & 61.46 & 6.02 & 12.50 \\
\hline 3 & Leaf Lnth (cm) & 11.52 & 17.21 & 14.90 & 2.14 & 2.37 & 9.82 & 10.33 & 90.42 & 2.87 & 19.24 \\
\hline 4 & Leaf Width $(\mathrm{cm})$ & 4.85 & 9.12 & 7.39 & 1.78 & 1.90 & 18.04 & 18.66 & 93.43 & 2.66 & 35.92 \\
\hline 5 & Petiol Lnth $(\mathrm{cm})$ & 5.15 & 95 & 6.27 & 0.34 & 0.48 & 9.30 & 10.99 & 71.58 & 1.02 & 16.20 \\
\hline 6 & Node No. & 55.00 & 112.00 & 88.50 & 181.85 & 201.37 & 15.24 & 16.03 & 90.31 & 26.40 & 29.83 \\
\hline 7 & Base Dia (mm) & 11.25 & 17.85 & 15.55 & 2.75 & 2.96 & 10.66 & 11.06 & 92.94 & 3.29 & 21.18 \\
\hline 8 & Middle Dia (mm) & 6.96 & 11.03 & 8.72 & 0.84 & 0.92 & 10.53 & 10.99 & 91.83 & 1.81 & 20.78 \\
\hline 9 & Top Dia (mm) & 3.28 & 5.92 & 4.29 & 0.35 & 0.38 & 13.70 & 14.45 & 89.84 & 1.15 & 26.75 \\
\hline 10 & Core Dia (mm) & 10.75 & 15.96 & 14.00 & 1.68 & 1.83 & 9.25 & 9.67 & 91.54 & 2.55 & 18.24 \\
\hline 11 & Dry Fibre wt. (g) & 5.89 & 11.93 & 9.80 & 2.39 & 2.49 & 15.79 & 16.11 & 96.11 & 3.13 & 31.89 \\
\hline 12 & Dry Stick wt. (g) & 15.25 & 33.87 & 26.98 & 20.96 & 21.38 & 16.97 & 17.14 & 98.06 & 9.34 & 34.62 \\
\hline
\end{tabular}

Table 4: Variability, heritability (h2b), genetic advance (GA) and GA in percent of mean for twelve yield and its related characters of C. olitorius. 
All genotypes were distributed in distinct divergent clusters. The distribution of the tossa jute germplasm accessions exhibiting higher fibre yield along with the different morpho agronomic factors in the five groups of divergent clusters are presented in table 5. In the first group of divergent clusters consisting of cluster-I, ten genotypes having higher average rank namely serial no. of Ac 01, Ac 05, Ac 07, Ac 18, Ac 25, Ac 26, Ac 27, Ac 33, Ac 37 and Ac 42 belonged to cluster-I. In the second group of divergent clusters consisting of cluster-II, sixteen genotypes namely serial no. of Ac 02, Ac 03, Ac 08, Ac 09, Ac 12, Ac 13, Ac 16, Ac 20, Ac 21, Ac 22, Ac 23, Ac 29, Ac 32, Ac 36, Ac 39 and Ac 40 belonged to cluster-II. In the third group of divergent clusters consisting of cluster-III, nine genotypes namely serial no. of Ac 04, Ac 11, Ac 17, Ac 30, Ac 31, Ac 38, Ac 41, Ac 43 and Ac 44 belonged to cluster-III. In the fourth group of divergent clusters consisting of cluster-IV, five genotypes namely serial no. of Ac 06, Ac 15, Ac 28, Ac 34 and Ac 35 belonged to cluster-IV. In the fifth group of divergent clusters consisting of cluster-V, four genotypes namely serial no. of Ac 10, Ac 14, Ac 19 and Ac 24 belonged to cluster-V.

\begin{tabular}{|c|c|c|c|}
\hline $\begin{array}{l}\text { Cluster } \\
\text { number }\end{array}$ & $\begin{array}{c}\text { Number of } \\
\text { varieties }\end{array}$ & $\begin{array}{c}\text { Percent } \\
(\%)\end{array}$ & Name of Accessions \\
\hline I & 10 & 22.73 & $\begin{array}{l}\text { Ac } 01, \text { Ac } 05, \text { Ac } 07, \text { Ac } \\
18, \text { Ac } 25 \text {, Ac 26, Ac 27, } \\
\text { Ac } 33 \text {, Ac } 37 \text { and Ac } 42\end{array}$ \\
\hline II & 16 & 36.36 & $\begin{array}{l}\text { Ac } 02, \text { Ac } 03, \text { Ac 08, Ac } \\
09, \text { Ac } 12 \text {, Ac } 13, \text { Ac } 16, \\
\text { Ac 20, Ac 21, Ac 22, Ac } \\
23, \text { Ac 29, Ac 32, Ac 36, } \\
\text { Ac } 39 \text { and Ac } 40\end{array}$ \\
\hline III & 9 & 20.45 & $\begin{array}{l}\text { Ac } 04, \text { Ac } 11, \text { Ac } 17, \text { Ac } \\
30 \text {, Ac } 31 \text {, Ac } 38 \text {, Ac } 41 \text {, } \\
\text { Ac } 43 \text { and Ac } 44\end{array}$ \\
\hline IV & 5 & 11.36 & $\begin{array}{c}\text { Ac 06, Ac } 15 \text {, Ac } 28 \text {, Ac } \\
34 \text { and Ac } 35\end{array}$ \\
\hline V & 4 & 9.09 & $\begin{array}{c}\text { Ac } 10 \text {, Ac } 14 \text {, Ac } 19 \text { and } \\
\text { Ac } 24\end{array}$ \\
\hline
\end{tabular}

Table 5: Number, percent and name of genotypes in different cluster.

The diversity in the present materials was also supported by the appreciable amount of variation among cluster means for different characters (Table 6). Cluster $\mathrm{V}$ showed highest mean for plant height $(3.73 \mathrm{~m})$, Base diameter $(17.51 \mathrm{~mm})$, dry stick weight (33.24g) and dry fibre weight (11.68g) [9].

\begin{tabular}{|l|c|c|c|c|c|}
\hline \multicolumn{1}{|c|}{ Characters } & I & II & III & IV & V \\
\hline Tech ht (m) & 2.91 & 3.22 & 2.62 & 3.54 & 3.73 \\
\hline Leaf Angl (dg) & 50.90 & 47.56 & 52.22 & 43.40 & 40.75 \\
\hline Leaf Lnth (cm) & 14.25 & 15.64 & 12.81 & 15.88 & 17.01 \\
\hline Leaf Width (cm) & 6.55 & 8.22 & 5.46 & 8.64 & 8.98 \\
\hline Petiol Lnth (cm) & 5.82 & 6.44 & 5.65 & 6.66 & 7.67 \\
\hline Node No. & 83.00 & 95.44 & 67.33 & 99.80 & 108.00 \\
\hline Base Dia (mm) & 15.14 & 16.34 & 12.88 & 17.05 & 17.51 \\
\hline $\begin{array}{l}\text { Middle Dia } \\
\text { (mm) }\end{array}$ & 8.45 & 8.90 & 7.62 & 9.53 & 10.16 \\
\hline Top Dia (mm) & 3.90 & 4.31 & 3.74 & 4.92 & 5.61 \\
\hline Core Dia (mm) & 13.51 & 14.76 & 12.04 & 14.73 & 15.66 \\
\hline $\begin{array}{l}\text { Dry Fibre wt. } \\
\text { (g) }\end{array}$ & 9.27 & 10.62 & 7.34 & 11.14 & 11.68 \\
\hline Dry Stick wt. (g) & 25.19 & 28.81 & 20.26 & 31.79 & 33.24 \\
\hline
\end{tabular}

Table 6: Cluster mean for twelve yield and yield characters of $\mathrm{C}$. olitorius.

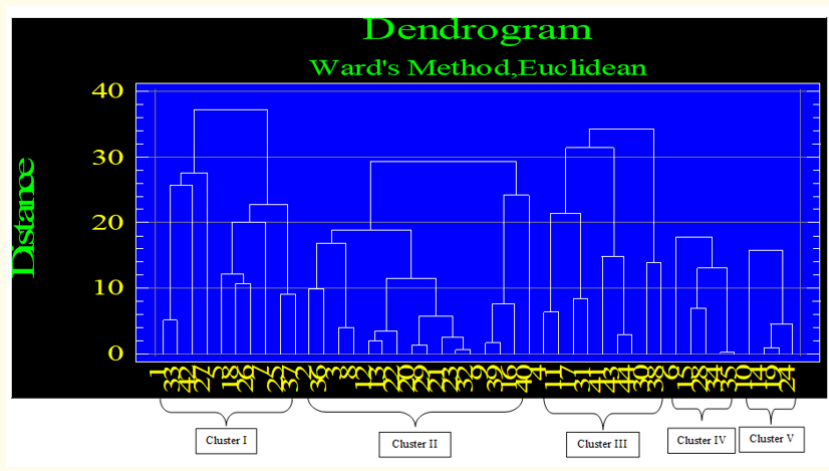

Figure 1: Structure of SARS CoV-2.

\section{Conclusion}

Significant ranges of variability were observed in plant height, base diameter, dry fibre weight, dry stick weight, green weight with leaves and fibre\%. These accessions 1039, 1115, 1124, 1234 and1245 performed better in most of the cases than the control varieties 0-9897 0-3820 and BJRI Tossa Pat-4 (0-72). Hence it can be concluded that these promising accessions may be used in a hybridization programme, to enhance fibre yield of tossa jute. 


\section{Bibliography}

1. Sawarkar A., et al. "Evaluation of quantitative traits of tossa jute (Corchorus olitorius l.) based on principal component analysis". Electronic Journal of Plant Breeding 6.1 (2015): 307311.

2. Islam S., et al. "Improved salt tolerance of jute plants expressing the kat E gene from Escherichia coli". Turkish Journal of Biology 37 (2013): 206-211.

3. Sarkar S. "Good practices for jute and allied fibre crops". In: Souvenir, International Symposium on jute and allied fibre Production, Utilization and Marketing. Organized by Indian Fibre Society (Eastern Region) (2008): 1-3.

4. Kumar D., et al. "Analysis for identification of distinct and uniform extant jute (Corchorus olitorius L. and C. capsularis L.) varieties". Seed Research 36 (2008): 121-134.

5. Roy SK., et al. "Studies on genetic variability and screening for fibre yield components and biotic stress factors in tossa jute (Corchorus olitorius L.) germplasm under Terai region of West Bengal". Electronic Journal of Plant Breeding 9.2 (2018): 409423.

6. Annual Research Report. "Bangladesh Jute Research Institute”. Manik Mia Avenue, Dhaka (2016): 1207.

7. Sawarkar A., et al. "Correlation and path coefficient analysis of yield and its attributing traits in tossa jute (Corchorus olitorius L.)". The Bioscan 9.2 (2014): 883-887.

8. Roy A., et al. "Exploiting plant growth promoting microbes and biopesticides for ecofriendly and cost effective biotic stress management of Olitorius Jute in Terai Region of West Bengal”. Indian Journal of Natural Fibres 1.2 (2015): 203-211.

9. Kenaf and Mesta crops. "Hand Book on Agricultural Technologies of Jute, Bangladesh Jute Research Institute". Manik Mia Avenue, Dhaka (2008):1207.

\section{Assets from publication with us}

- Prompt Acknowledgement after receiving the article

- Thorough Double blinded peer review

- Rapid Publication

- Issue of Publication Certificate

- High visibility of your Published work

Website: www.actascientific.com/

Submit Article: www.actascientific.com/submission.php Email us: editor@actascientific.com

Contact us: +919182824667 